\title{
A natação forçada induz subsensibilidade à fenilefrina em aorta torácica de rato
}

\author{
Maria José C. Sampaio Moura1, Maísa Momesso de Quintal', Fernanda Klein Marcondes* \\ 'Laboratório de Fisiologia, Faculdade de Ciências Biológicas, Centro de Ciências da Vida (CCV), PUC-Campinas, \\ ${ }^{2}$ Departamento de Ciências Fisiológicas, Faculdade de Odontologia de Piracicaba, UNICAMP
}

*Correspondência:

F. K. Marcondes

Depto. de Ciências Fisiológicas

Faculdade de Odontologia de

Piracicaba - FOP/UNICAMP

Av. Limeira, 901, 13414-903

Piracicaba/SP

E-mail: fklein@fop.unicamp.br
$O$ estresse pode alterar a função vascular. O objetivo deste trabalho foi estudar a sensibilidade à fenilefrina (FE) em aorta torácica de ratos submetidos à natação forçada. Ratos Wistar (200-250 g) foram submetidos a três sessões de natação aplicadas em dias consecutivos (15, 30 e 30 min respectivamente). Imediatamente após a última sessão, os animais foram sacrificados e, da aorta torácica foram obtidos anéis (3-5 $\mathrm{mm})$ com e sem endotélio. Os anéis foram mantidos em solução de Krebs-Henseleit $\left(37{ }^{\circ} \mathrm{C}\right.$; $\left.95 \% \mathrm{O}_{2}-5 \% \mathrm{CO}_{2}\right)$. A integridade do endotélio foi avaliada pelo relaxamento em resposta à acetilcolina $(10 \mu M)$, após indução de contração por FE $(0,1 \mu M)$. Curvas concentração-efeito à FE foram obtidas ( $n=5 /$ grupo). Não houve diferença na resposta máxima à $F E$ entre os tratamentos controle $e$ estresse, em anéis com e sem endotélio $(p>0,05)$. A natação forçada induziu subsensibilidade à FE em anéis com endotélio de aorta torácica isolada de ratos submetidos à natação $(\mathrm{pD}=$ $6,89 \pm 0,07, p<0,05)$, em relação ao grupo controle $\left(p D_{2}=7,46 \pm\right.$ 0,06), sem alteração nos anéis desprovidos de endotélio. A inibição in vitro da sintese de óxido nitrico cancelou esta subsensibilidade. Em conclusão, a subsensibilidade à FE induzida pela natação forçada parece ser causada pelo aumento da atividade do sistema de óxido nitrico endotelial.

\section{INTRODUÇÃO}

A reação de estresse compreende o conjunto de respostas do organismo a agentes aversivos ou desconhecidos, externos ou internos, as quais desencadeiam processos adaptativos para a manutenção de sua homeostasia (Cannon et al., 1927; Selye, 1936). Os principais media- dores da resposta ao estresse são as catecolaminas liberadas pelo sistema nervoso simpático e pela medula da adrenal, e os glicocorticóides liberados pelo córtex da adrenal (McEwen, 2000).

$\mathrm{Na}$ tentativa de compreender as alterações fisiológicas que ocorrem durante a reação de estresse, diferentes modelos experimentais têm sido utilizados em estudos de 
laboratório, tais como imobilização (Capaz, De Moraes, 1988; Harri et al., 1974), choques nas patas (Bassani, De Moraes, 1987; Marcondes et al., 1996), exposição a frio (Harri et al., 1974; Callia, De Moraes, 1984) e natação (Moura, De Moraes, 1994; Bianchi et al., 2001; Tanno et al., 2002 a.,b). Os resultados destes estudos mostram diferentes efeitos do estresse sobre a sensibilidade do sistema cardiovascular às ações das catecolaminas, sendo tais efeitos dependentes da qualidade, intensidade e frequência do agente estressor.

A exposição de ratos ao frio induziu subsensibilidade ao efeito cronotrópico da norepinefrina, decorrente de uma alteração na estrutura dos adrenoceptores $\beta_{1}$ cardíacos (Callia, De Moraes, 1984). Foi observada subsensibilidade aos efeitos cronotrópicos da norepinefrina e da isoprenalina em marcapasso isolado de ratos submetidos à imobilização (Capaz, De Moraes, 1988) ou a três sessões de natação em espaço amplo (Spadari, De Moraes, 1988). Por outro lado, a aplicação de sessão única, em espaço amplo (Spadari et al., 1988; Tanno et al., 2002 a,b), ou de sessões repetidas de natação em espaço restrito (Moura, De Moraes, 1994), induziu supersensibilidade ao efeito cronotrópico da isoprenalina em átrio direito isolado de ratos. Estes efeitos do estresse sobre o controle adrenérgico da resposta cronotrópica depende, pelo menos parcialmente, dos níveis plasmáticos de corticosterona (Spadari, De Moraes, 1988; Capaz, De Moraes, 1988; Nourani et al., 1992; Moura, De Moraes, 1994).

Também foram observadas alterações da sensibilidade vascular às catecolaminas in vitro. $\mathrm{O}$ frio reduziu a sensibilidade da artéria carótida de coelhos ao efeito vasoconstritor da norepinefrina (Flain, Hsieh, 1978). O estresse por imobilização induziu diminuição da resposta ao efeito vasoconstritor da norepinefrina em aorta torácica de ratos (Cordellini, Vassilieff, 1998). Martins e Spadari (1990) observaram subsensibilidade in vivo ao efeito pressor da norepinefrina em ratos submetidos a uma sessão de natação. As autoras sugeriram que a subsensibilidade à catecolamina decorria de uma reduzida participação dos adrenoceptores alfa vasculares na ação vasoconstritora da norepinefrina.

As alterações nas respostas às catecolaminas podem envolver a modulação da atividade de adrenoceptores alfa ou beta, e alterações nos processos de metabolização das catecolaminas (Tanno et al., 2002a, b). O uso de agonistas seletivos e sintéticos, que não sejam substrato dos processos de metabolização das catecolaminas, constitui uma ferramenta para o estudo dos mecanismos envolvidos nas alterações de sensibilidade adrenérgica. O objetivo deste trabalho foi estudar a sensibilidade alfa-adrenérgica ao efeito contrátil da fenilefrina (FE) em aorta torácica de ratos submetidos à natação forçada e avaliar a participação do endotélio na modulação da atividade contrátil deste tecido após a natação forçada.

\section{MATERIAL E MÉTODOS}

Foram utilizados ratos Wistar, machos, pesando entre 200 e $250 \mathrm{~g}$. Os animais foram mantidos, em grupo de três, em gaiolas plásticas $(30 \times 16 \times 19 \mathrm{~cm})$, recebendo água e ração à vontade, durante uma semana antes de qualquer manipulação experimental. Todos os procedimentos foram aprovados pelo Comitê de Ética em Experimentação Animal do Instituto de Biologia da Universidade Estadual de Campinas, de acordo com as normas do Colégio Brasileiro de Experimentação Animal (COBEA).

Os animais foram submetidos, individualmente, à natação forçada em um tanque cilíndrico de PVC, com diâmetro de $22 \mathrm{~cm}$ (Porsolt et al., 1978; Moura, De Moraes, 1994). A altura da coluna de água foi de $35-38 \mathrm{~cm}$, suficiente para evitar que os animais encostassem as patas ou a cauda no fundo do tanque. As sessões de natação foram aplicadas, entre 8 e $11 \mathrm{~h}$, em dias consecutivos, com duração de 15,15 e 30 min, respectivamente no primeiro, segundo e terceiro dias. Após a primeira e a segunda sessões de natação, os animais foram mantidos próximos a um aquecedor de ambiente, retornando ao biotério após estarem completamente secos. Imediatamente após a terceira sessão de natação, os animais foram sacrificados por concussão cerebral seguida de secção dos vasos cervicais (Moura, De Moraes, 1994). Ratos do grupo controle, submetidos somente à manipulação relativa à limpeza das gaiolas, também foram sacrificados da mesma maneira.

A aorta torácica de cada animal foi cuidadosamente isolada para preservação do endotélio. De cada aorta foram retirados dois anéis $(3-5 \mathrm{~mm})$, tendo um deles sua superfície interna delicadamente massageada para remoção do endotélio. Os anéis foram montados sob $2 \mathrm{~g}$ de tensão inicial, em câmara para órgão isolado contendo solução de Krebs-Henseleit com a seguinte composição (mM): $\mathrm{NaCl}, 115,0 ; \mathrm{KCl}, 4,6 ; \mathrm{CaCl}_{2} 2 \mathrm{H}_{2} \mathrm{O}, 2,5 ; \mathrm{KH}_{2} \mathrm{PO}_{4}, 1,2$; $\mathrm{MgSO}_{4} 7 \mathrm{H}_{2} \mathrm{O}, 2,5 ; \mathrm{NaHCO}_{3}, 25,0$ e glicose 11,0. O líquido de incubação foi continuamente borbulhado com $5 \%$ de $\mathrm{CO}_{2}$ e $95 \%$ de $\mathrm{O}_{2}$ e mantido a $37^{\circ} \mathrm{C}$.

Antes da adição de qualquer droga, os anéis permaneceram no banho para equilíbrio, por 60 minutos, e a solução de Krebs foi trocada a cada $15 \mathrm{~min}$. Após o equilíbrio, a presença do endotélio foi comprovada fisiologicamente pela indução de relaxamento com a adição de acetilcolina $(10 \mu \mathrm{M})$ em anéis pré-contraídos com fenilefrina $(0,1 \mu \mathrm{M})$. Considerou-se que o endotélio estava presente nos anéis que apresentaram no mínimo $80 \%$ de relaxamento e ausente 
nos anéis que não apresentaram nenhum relaxamento. Anéis que apresentaram relaxamento inferior a $80 \%$, em resposta à acetilcolina, foram descartados. Após este teste, os anéis foram reequilibrados por um período de $45 \mathrm{~min}$, com troca de Krebs a cada 15 min (Moura, Marcondes, 2001).

Após o período de reequilíbrio acima descrito, uma curva concentração-efeito cumulativa (Van Rossum, 1963) à fenilefrina foi obtida em cada anel. Os incrementos da concentração molar foram de 0,5 unidade logarítmica. $\mathrm{O}$ efeito máximo foi determinado quando três concentrações sucessivas e crescentes do agonista não alteraram a resposta obtida com a concentração imediatamente anterior. As respostas, transformadas em gf/100mg de tecido, foram expressas em porcentagens das respostas máximas. As curvas concentração-efeito foram analisadas por regressão não linear (Moura, Marcondes, 2001). A sensibilidade da aorta foi avaliada pela determinação do valor $\mathrm{pD}_{2}$, que corresponde ao logaritmo negativo da concentração molar do agonista $\left(\mathrm{EC}_{50}\right)$ que determina um efeito igual a $50 \%$ da resposta máxima em experimentos individuais.

Para a avaliação do papel do óxido nítrico (NO), derivado do endotélio, na modulação da sensibilidade à FE, anéis com endotélio íntegro obtidos de aorta isolada de outros animais controle ou submetidos à natação forçada, foram incubados por $40 \mathrm{~min}$ com o inibidor da síntese de $\mathrm{NO}, \mathrm{N}^{\mathrm{G}}$-L-Arginina-metil-ester (L-NAME, $10^{-5} \mathrm{M}$ ), após a verificação da presença do endotélio, conforme descrição acima. Após o período de reequilíbrio, uma curva concentração-efeito à fenilefrina foi obtida em cada anel.

Os resultados são apresentados como médias acompanhadas de seus respectivos erros-padrão (EPM). Diferenças, nos valores de $\mathrm{RM}$ (resposta máxima) e $\mathrm{pD}_{2}$, entre os tratamentos estresse e controle foram avaliadas pelo teste $t$ de Student para amostras não pareadas. Valores de $\mathrm{p}$ menores do que 0,05 foram indicativos de significância estatística.

\section{RESULTADOS}

Não houve diferença nos valores de resposta máxima à fenilefrina entre os grupos estudados, em anéis com ou sem endotélio (Tabela I; $p>0,05$ ). Assim sendo, as curvas concentração-efeito estão apresentadas em porcentagem da resposta máxima. Em anéis da aorta torácica, com endotélio íntegro, foi observada diminuição de sensibilidade à fenilefrina no tecido isolado de animais submetidos à natação forçada, em relação ao grupo controle (Figura 1; $\mathrm{p}=$ 0,0002 ). Esta subsensibilidade foi caracterizada por um desvio significativo de 4 vezes à direita na curva concentração-efeito à fenilefrina no tecido de animais submetidos a estresse quando comparada à curva obtida no tecido isola- do de animais controle (Figura 1). Em anéis da aorta torácica, desprovidos de endotélio, não houve diferença de sensibilidade à fenilefrina entre animais controles e estressados (Figura 2, p =0,9034). Não houve diferença entre os grupos controle e submetido à natação forçada, nas curvas concentração-efeito à fenilefrina, obtidas na presença do inibidor da síntese de óxido nítrico, L-NAME (Figura $3, p=0,4264)$.

TABELA I - Valores de resposta máxima (RM) à fenilefrina em anéis, com e sem endotélio, de aorta torácica isolada de ratos controle ou submetidos à natação forçada

\begin{tabular}{ccc}
\hline Grupos & $\mathbf{N}^{\mathbf{a}}$ & $\begin{array}{c}\mathbf{R M}^{\mathbf{b}} \\
(\mathbf{g f} / \mathbf{1 0 0} \mathbf{~ m g})\end{array}$ \\
\hline Anéis com endotélio & & \\
$\quad$ Controle & 5 & $36 \pm 5$ \\
$\quad$ Natação & 5 & $36 \pm 9$ \\
Anéis sem endotélio & & \\
$\quad$ Controle & 5 & $27 \pm 4$ \\
$\quad$ Natação & 5 & $36 \pm 6$ \\
Anéis incubados com L-NAMEc & \\
$\quad$ Controle & 5 & $35 \pm 9$ \\
$\quad$ Natação & 5 & $34 \pm 7$ \\
\hline
\end{tabular}

${ }^{a}$ Número de experimentos. ${ }^{b}$ Valores médios \pm errospadrão das médias. ${ }^{\mathrm{c}} \mathrm{L}-\mathrm{NAME}=$ éster metílico da $N^{\mathrm{g}}-\mathrm{L}-$ Arginina, inibidor da síntese de óxido nítrico.

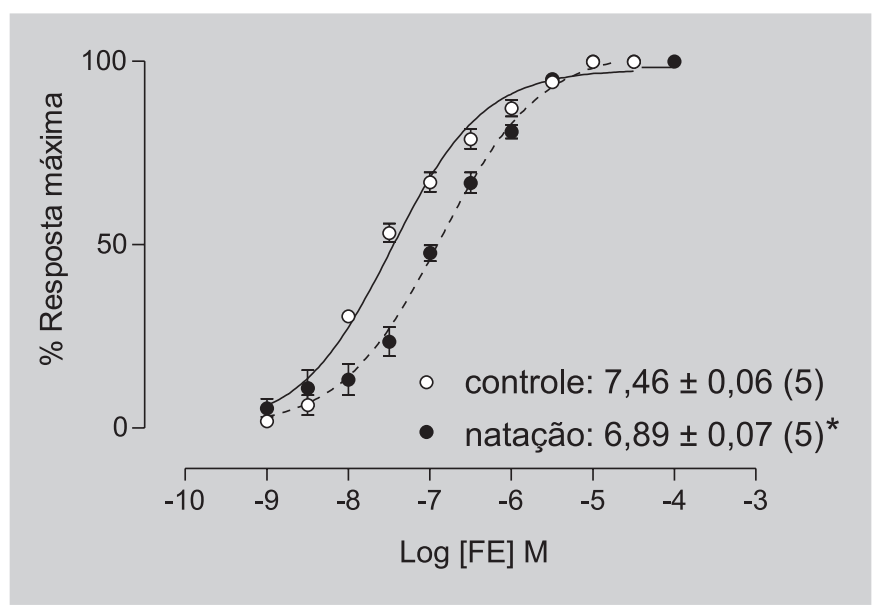

FIGURA 1 - Curvas concentração-efeito à fenilefrina em anéis com endotélio, isolados da aorta torácica de ratos controle ou submetidos à natação forçada. Os valores indicados na legenda representam a média \pm erro-padrão da média dos logaritmos negativos da concentração molar de fenilefrina que determinou $50 \%$ da resposta máxima nos tecidos isolados (n). *Estatisticamente diferente do grupo controle $(\mathrm{p}<0,05)$. 


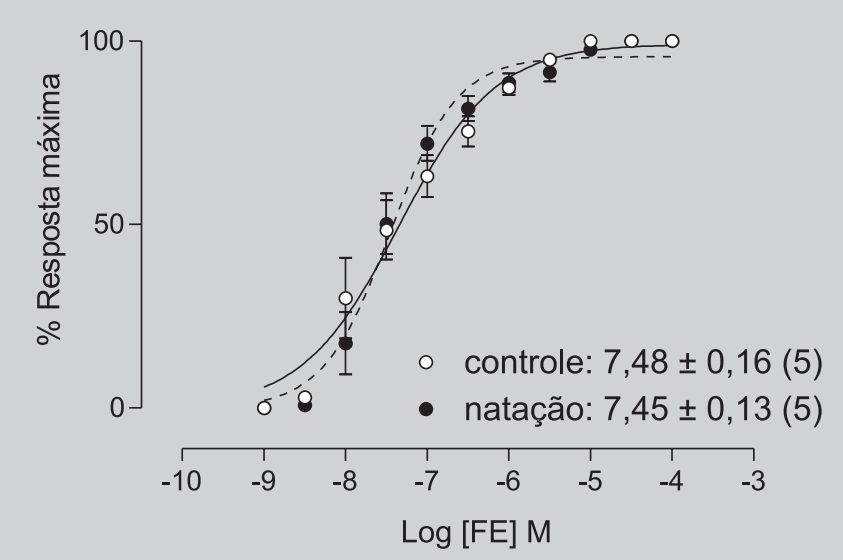

FIGURA 2 - Curvas concentração-efeito à fenilefrina em anéis sem endotélio, isolados da aorta torácica de ratos controle ou submetidos à natação forçada. Os valores indicados na legenda representam a média \pm erro-padrão da média dos logaritmos negativos da concentração molar de fenilefrina que determinou $50 \%$ da resposta máxima nos tecidos isolados (n).

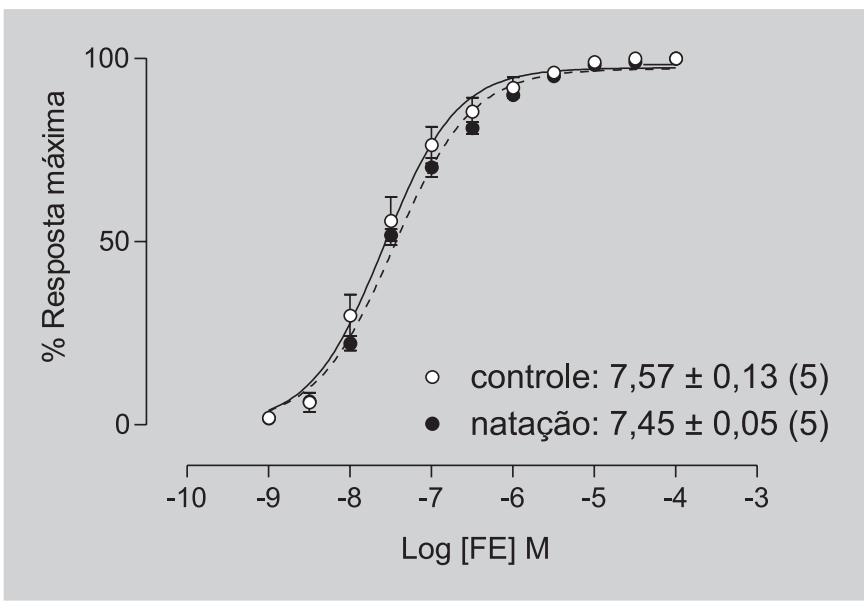

FIGURA 3 - Curvas concentração-efeito à fenilefrina obtidas, na presença do inibidor da síntese de óxido nítrico L-NAME, em anéis isolados da aorta torácica de ratos controle ou submetidos à natação forçada. Os valores indicados na legenda representam a média \pm erro-padrão da média dos logaritmos negativos da concentração molar de fenilefrina que determinou $50 \%$ da resposta máxima nos tecidos isolados (n).

\section{DISCUSSÃO}

A natação é um dos protocolos experimentais utilizados em estudos sobre estresse. Além de representar um agente estressor por seu componente físico, a natação apresenta um forte componente emocional, relacionado à novidade que este estímulo representa para o animal e à impossibilidade de fuga, somada à iminência de morte (Östman-Smith, 1979; Garcia-Marques, Armário, 1987; Marcondes et al., 1996; Bianchi et al., 2001). Porém, diferentes protocolos experimentais de natação têm sido utilizados resultando em diferentes respostas em nível celular, entre as quais merecem destaque as alterações de sensibilidade às catecolaminas em tecidos periféricos. As diferenças entre os resultados obtidos estão relacionadas à intensidade dos componentes físico e emocional envolvidos na adaptação ao estresse.

Neste trabalho, ratos foram submetidos à natação forçada em espaço restrito, em três dias consecutivos. Em anéis, com endotélio intacto, de aorta torácica isolada de animais submetidos a este modelo experimental houve diminuição de sensibilidade à fenilefrina em relação ao tecido isolado de animais controle.

Jansakul (1995) mostrou que há uma diminuição na reatividade à fenilefrina, mas não ao $\mathrm{KCl}$, em anéis de aorta torácica de ratos submetidos à natação durante 5 a 6 semanas. Oltman et al. (1992) mostraram resultados semelhantes em suínos. Por outro lado, o treinamento físico induziu subsensibilidade à norepinefrina e ao $\mathrm{KCl}$, sem alteração da resposta vascular à fenilefrina (Delp et al., 1993). Também Spier et al. (1999) relataram o desenvolvimento de subsensibilidade à norepinefrina em ratos treinados, por 10 semanas, em esteira. Entretanto, em cães, a resposta contrátil de artérias coronárias à norepinefrina e fenilefrina não foram diferentes entre animais treinados e sedentários (Rogers et al., 1991). Também, não foram observadas alterações de sensibilidade à norepinefrina em tiras helicoidais de aortas de ratos treinados (Edwards et al., 1985).

Estas diferenças poderiam ser atribuídas às diferenças nas espécies animais estudadas, às diferenças nos tecidos vasculares utilizados e ao tipo de preparação. O mecanismo do efeito vasoconstritor da norepinefrina é diferente na aorta torácica e abdominal sendo nesta última, mediada em parte, por um metabólito da cicloxigenase (Lamb et al. 1994). Com relação ao tipo de preparação utilizada, Jansakul (1995) trabalhou com anéis enquanto que Edwards et al. (1985) utilizaram tiras helicoidais de aortas de ratos.

Considerando que a fenilefrina não é substrato dos sistemas de metabolização das catecolaminas, a subsensibilidade, induzida pela natação observada no presente estudo, não parece envolver alteração nos processos de recaptação neuronal e captação extraneuronal, como já foi demonstrado em outros tecidos (Spadari, De Moraes, 1988). A ausência de subsensibilidade nos anéis desprovidos de endotélio sugere que a alteração observada deve-se 
a alguma alteração na função endotelial e não à ação direta do estresse sobre a musculatura lisa vascular. Assim, esta menor sensibilidade à fenilefrina pode estar relacionada a processos compensatórios como a diminuição da densida-

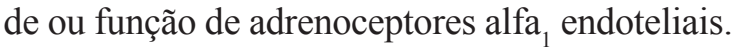

O endotélio vascular foi considerado durante muito tempo como um conjunto de células cuja função primária seria a de revestir internamente os vasos sanguíneos, separando os espaços intravascular e extravascular, garantindo assim uma superfície lisa para o fluxo sanguíneo. $\mathrm{O}$ clássico experimento de Furchgott (Furchgott, Zawadski, 1980) estabeleceu o papel fundamental do endotélio na modulação da atividade contrátil vascular através da produção e liberação de um fator relaxante derivado do endotélio, posteriormente identificado como óxido nítrico (Palmer et al. 1987; Moncada, 1991). Além do óxido nítrico, a prostaciclina (Gryglewski et al., 1988) e um fator hiperpolarizante (Feletou, Vanhoutte, 1976), ambos derivados do endotélio, determinam o relaxamento da musculatura lisa vascular. Este tecido pode ainda liberar substâncias ou fatores que determinam vasoconstrição e que foram, coletivamente, denominados de vasoconstritores derivados do endotélio (Rubanyi, Vanhoutte, 1985; Yanagisawa et al., 1988; De Moraes et al., 1995).

A importância da participação do endotélio em processos fisiopatológicos vasculares é indiscutível. Neste contexto, investigamos no presente estudo, sua participação na diminuição da sensibilidade à fenilefrina em aortas torácicas com endotélio intacto de ratos estressados. Os resultados obtidos mostraram que este efeito foi cancelado pela inibição da síntese de óxido nítrico. Portanto, estes dados indicam que a natação forçada parece ter induzido um aumento na síntese de óxido nítrico na aorta torácica. Respostas semelhantes foram induzidas por outros protocolos experimentais.

O estresse agudo e crônico por imobilização promoveu o aumento na liberação de óxido nítrico, pelo endotélio vascular, o que atenuou a resposta à fenilefrina (Cordellini; Vassilieff, 1998; Navarro-Oliveira et al., 2000). Também foi relatado que o exercício físico por natação em espaço amplo induziu diminuição da resposta vasoconstritora à fenilefrina através do aumento na liberação de óxido nítrico derivado de endotélio (Jansakul, 1995).

O sistema vascular tem um papel crítico no desenvolvimento de alterações funcionais e estruturais responsáveis por danos a órgãos e tecidos em animais de laboratório e na espécie humana, em patologias, envelhecimento e em resposta ao estresse (Plante, 2002). Neste contexto, nossos resultados evidenciam a importância dos estudos sobre a resposta vascular a agonistas adrenérgicos em situações de estresse, tendo em vista que alterações nos adrenoceptores endoteliais ou do músculo liso vascular podem modificar a resposta a fármacos utilizados durante situações estressantes e/ou tratamento de doenças cardiovasculares.

\section{ABSTRACT \\ Forced-swim induces subsensitivity to phenylephrine in the rat thoracic aorta.}

Stress may change vascular function. The aim of this report was to study the sensitivity to phenylephrine (PHE) in the thoracic aorta from rats submitted to forced-swim. Male Wistar rats (200-250 g) were submitted to three swimming sessions, one session/day (15, 30 and $30 \mathrm{~min}$, respectively). Immediately after the last swimming session, the animals were sacrificed and thoracic aorta was isolated. Aortic rings (3-5 $\mathrm{mm})$, with and without endothelium, were carefully obtained and were maintained in Krebs-Henseleit solution $\left(95 \% \mathrm{O}_{2}-5 \% \mathrm{CO}_{2}\right.$, $37^{\circ} \mathrm{C}$ ). Endothelial integrity was assessed by relaxation to acetylcholine $(10 \mu \mathrm{M})$ in pre-contracted rings (PHE $0.1 \mu \mathrm{M})$. Concentration-effect curves to PHE were obtained ( $n=5 /$ group). There was no difference between control and stress groups in the maximum response to PHE of aortic rings with and without endothelium $(p>0.05)$. Forced-swim induced subsensitivity to PHE in aortic rings with endothelium isolated from stressed rats $\left(p D_{2}=6.89 \pm 0.07 ; p<0.05\right)$ compared to the control group $\left(p D_{2}=7.39 \pm 0.06\right)$, without changes in aortic rings without endothelium. The in vitro inhibition of nitric oxide synthesis cancelled this subsensitivity. It is concluded that forced swim-induced-subsensitivity to PHE in thoracic aorta from rats seems to be caused by an increase in the activity of the endothelial nitric oxide system.

UNITERMS: Stress. Phenylephrine. Subsensitivity. Aorta. Endothelium. Swimming.

\section{AGRADECIMENTOS}

Este estudo recebeu auxílio financeiro do Fundo de Apoio ao Ensino e Pesquisa da Universidade Estadual de Campinas (FAEP/UNICAMP).

\section{REFERÊNCIAS BIBLIOGRÁFICAS}

BASSANI, R. A.; DE MORAES, S. Subsensitivity to beta adrenoceptor agonists in right atria isolated from footshock stressed rats. Gen. Pharmacol., Oxford, v. 18, n. 5 , p. 473-477, 1987. 
BIANCHI, F. J.; TANNO, A. P.; MARCONDES, F.K. Relação entre nível de estresse e supersensibilidade à norepinefrina em ratas no proestro. Rev. Bras. Ciênc. Farm., São Paulo, v. 37, n. 3, 391-398, 2001.

CALLIA, M. L.; DE MORAES, S. Heterogeneity of beta adrenoceptors in right atria isolated from cold-exposed rats. J. Pharmacol. Exp. Ther., Baltimore, v. 230, n. 2, p. 450-454, 1984.

CANNON, W.B.; QUERIDO, S.; BRITTON, S.W.; BRIGHT, E.M. Studies on the conditions of activity in endocrine glands. The role of adrenal excretion in the chemical control of body temperature. Am. J. Physiol., Bethesda, v. 79, p. 466-506, 1927.

CAPAZ, F. R.; DE MORAES, S. Reduction by acute restraint stress of norepinephrine sensitivity in the isolated rat pacemaker. Eur. J. Pharmacol., Amsterdam, v. 147, n. 2 , p. 295-298, 1988.

CORDELLINI, S.; VASSILIEFF, V.S. Decreased endothelium-dependent vasoconstriction to noradrenaline in acute-stressed rats is potentiated by previous chronic stress: nitric oxide involvement. Gen. Pharmacol., Oxford, v. 30, p.79-83, 1998.

DELP, M.D.; McALLISTER, R.M.; LAUGHLIN, M.H. Exercise training alters endothelium-dependent vasoreactivity of rat abdominal aorta. J. Appl. Physiol., Bethesda, v. 75, p. 1354-1363, 1993.

DE MORAES, S.; CARVALHO, A.J.; CAVALCANTE, M.T.; MATHIAS, S.R. Relative hypoxia-induced contraction of the isolated human umbilical artery. Pharmacol. \& Toxicol., Copenhagen, v. 76, p. 218-220, 1995.

EDWARDS, J. G.; TIPTON, C. M.; MATTHES, R. D. Influence of exercise training on reactivity and contractility of arterial strips from hypertensive rats. $J$. Appl. Physiol., Bethesda, v. 58, p. 1683-1688, 1985.

FELETOU, M.W.; VANHOUTTE, P.M. Endotheliumdependent hyperpolarization of canine coronary smooth muscle. Br. J. Pharmacol., London, v. 93, p. 515-524, 1976.

FLAIN, S.F.; HSIEH, A.C.L. Effect of cold-acclimation on rabbit carotid artery: altered response to norepinephrine. Gen. Pharmacol., Oxford, v.78, p. 437-442, 1978.
FURCHGOTT, R.F.; ZAWADZKI, J. V. The obligatory role of endothelial cells in the relaxation of arterial smooth muscle by acetylcholine. Nature, London, v. 288, p. 373-376, 1980.

GARCIA-MARQUEZ, C. G.; ARMARIO, A. Chronic stress depresses exploratory activity and behavioral performance in the forced swimming test without altering ACTH response to a novel acute stressor. Physiol. Behav., New York, v. 40, p. 33-38, 1987.

GRYGLEWSKI, R.J.; BOTTING, R. M.; VANE, J. R. Mediators produced by endothelial cells. Hypertension, Philadelphia, v. 12, p.530-548, 1988.

HARRI, M. N. E.; MELENDER, L.; TIRRI, R. Changed chronotropic sensitivity to sympathomimetic amines in isolated atria from rats following cold acclimation. Experientia, Basel, v. 30, n. 9, p. 1041-1043, 1974.

JANSAKUL, C. Effect of swimming on vascular reactivity to phenylephrine and $\mathrm{KCl}$ in male rats. Br. J. Pharmacol., London, v. 115, p. 587-594, 1995.

LAMB, V.L.; SCHWARTZ, A.; ROHN, W.R.; KAISER, L. Ciclooxigenase inhibitors depress norepinephrine constriction of rat abdominal, but not thoracic, aorta. Eur. J. Pharmacol., Amsterdam, v. 256, p. 221-226, 1994.

MARCONDES, F. K.; VANDERLEI, L. C. M.; LANZA, L. L. B.; SPADARI-BRATFISCH, R. C. Stress-induced subsensitivity to catecholamines depends on the estrous cycle. Can. J. Physiol. Pharmacol., Ottawa, v. 74, n. 6, p. 663-669, 1996.

MARTINS, M.C.; SPADARI, R.C. Stress-induced desensitization of the cardiovascular response to noradrenaline in unanesthetized rats. Braz. J. Med. Biol. Res., São Paulo, v. 23, p. 1041-1044, 1990.

McEWEN, B. S. Definitions and Concepts of Stress. In: FINK, G., ed. Encyclopedia of Stress. USA: Academic Press, 2000. v. 3, p. 508-509.

MONCADA, S.; PALMER, R.M.J.; HIGGS, E.A. Nitric oxide: physiology, pathology and pharmacology. Pharmacol. Rev., Bethesda, v. 43, p. 109-142, 1991.

MOURA, M.J.C.S.; DE MORAES, S. Forced swim stress: supersensitivity of the isolated rat pacemaker to the chronotropic effect of isoprenaline and the role of corticosterone. Gen. Pharmacol., Oxford, v. 25, p. 13411347, 1994. 
MOURA, M. J. C. S.; MARCONDES, F. K. Influence of estradiol and progesterone on the sensitivity of rat thoracic aorta to noradrenaline. Life Sci., Oxford, v. 68, n. 8, p. 881-888, 2001.

NAVARRO-OLIVEIRA, C.M.; VASSILIEFF, V.S.; CORDELLINI, S. The sympathetic adrenomedullary system, but not the hypothalamic-pituitary-adrenal axis, participates in aorta adaptive response to stress: nitric oxide involvement. Auton. Neurosci., Amsterdam, v. 83, n. 3, p. 140-147, 2000.

NOURANI, F.R.R.; SPADARI, R.C.; DE MORAES, S. Footshock stress-induced supersensitivity to isoprenaline in the isolated pacemaker of the rat: effects of the compounds RU-38486 and RU-28362. Gen. Pharmacol., Oxford, v. 23, p. 787-791, 1992.

PALMER, R.M.J.; FERRIDGE, A.C.; MONCADA, S. Nitric oxide release accounts for the biological activity of endothelium-derived relaxing factor. Nature, London, v. 327, p. 524-526, 1987.

OLTMAN, C. L.; PARKER, J. L.; ADAMS, H. R.; LAUGHLIN, M. H. Effects of exercise training on vasomotor reactivity of porcine coronary arteries. Am. J. Physiol., Bethesda, v.263, p. H372-H382, 1992.

ÖSTMAN-SMITH, I. Adaptive changes in the sympathetic nervous system and some effector organs of the rat following long-term exercise or cold acclimation and the role of cardiac sympathetic nerves in the genesis of compensatory cardiac hypertrophy. Acta Physiol. Scand., Oxford, suppl. 477, p. 1-118, 1979.

PLANTE, G.E. Vascular response to stress in health and disease. Metabolism, Bethesda, v. 51, n. 6, suppl. 1, p. 2530, 2002.

PORSOLT, R.D.; ANTON, G.; BLAVET, N.; JALFRE, M. Behavioral despair in rats: a new model sensitive to antidepressant treatments. Eur. J. Pharmacol., Amsterdam, v. 47, p. 379-391, 1978.

ROGERS, P. J.; MILLER, T. D.; BAUER, B. A.; BRUM, J. M.; BOVE, A. A.; VANHOUTTE, P. M. Exercise training and responsiveness of isolated coronary arteries. J. Appl. Physiol., Bethesda, v. 71, p. 2346-2351, 1991.
RUBANYI, G.M.; VANHOUTTE, P.N. Hypoxia releases a vasoconstrictor substance from canine vascular endothelium. J. Physiol., London, v. 363, p. 45-56, 1985.

SELYE, H. A syndrome produced by diverse nocuous agents. Nature, London, v. 138, n. 1, p. 32, 1936.

SPADARI, R. C.; DE MORAES, S. Repeated swimming stress and responsiveness of isolated rat pacemaker to chronotropic effect of noradrenaline and isoprenaline: role of adrenal corticosteroids. Gen. Pharmacol., Oxford, v. 19, n. 4 , p. $553-557,1988$.

SPADARI, R. C.; BASSANI, R. A.; DE MORAES, S. Supersensitivity to isoprenaline and epinephrine in right atria isolated from rats submitted to a single swimming session. Gen. Pharmacol., Oxford, v. 19, n. 1, p. 129-135, 1988.

SPIER, S.A.; LAUGHLIN, M.H.; DELP, M.D. Effects of acute and chronic exercise on vasoconstrictor responsiveness of rat abdominal aorta. J. Appl. Physiol., Bethesda, v. 87, p. 1752-1757, 1999.

TANNO, A.P.; BIANCHI, F. J.; MARCONDES, F.K. Influência do ciclo estral sobre a sensibilidade da resposta cronotrópica à norepinefrina em ratas submetidas a estresse agudo. Rev. Bras. Ciênc. Farm., São Paulo, v.38, n.1, p. 63-70, 2002a.

TANNO, A.P.; BIANCHI, F. J.; MOURA, M. J. C. S.; MARCONDES, F.K. Atrial supersensitivity to noradrenaline in stressed female rats. Life Sci., Oxford, v.71, n. 25, p. 2973-981, 2002b.

VAN ROSSUM, J. M. Cumulative dose-response curves. II. Technique for the making of dose-response curves in isolated organs and the evaluation of the drug parameters. Arch. Int. Pharmacodyn. Ther., Ghent, v. 143, p. 229-230, 1963.

YANAGISAWA, M.; KURIHARA, H.; KIMURA, S.; TOMOBE, Y.; KOBAYASHI, M.; MITSUE, Y.; YASAKI, Y.; GOTO, K.; MASAKI, T. A novel potent vasoconstrictor peptide produced by vascular endothelial cells. Nature, London, v. 332, p. 411-415, 1988.

Recebido para publicação em 15 de abril de 2003. 